\title{
Exaggerated Interventricular Dependence Among Patients With Pectus Excavatum: Combined Assessment With Cardiac MRI and Chest CT
}

\author{
Alejandro Deviggiano ${ }^{1}$ \\ Javier Vallejos ${ }^{1}$ \\ Natalia Vina ${ }^{1}$ \\ Marcelo Martinez-Ferro² \\ Gaston Bellia-Munzon² \\ Patricia Carrascosa ${ }^{1}$ \\ Gaston A. Rodríguez-Granillo ${ }^{1}$
}

Keywords: chest, CT, MRI, thoracic malformation, thoracic surgery

DOI:10.2214/AJR.16.17296

Received August 22, 2016; accepted without revision October 10, 2016.

P. Carrascosa is a consultant for GE Healthcare.

'Department of Computed Tomography and Magnetic Resonance Imaging, Diagnóstico Maipu, Avenue Maipu 1668, Buenos Aires, Vicente López B1602B0, Argentina. Address correspondence to G. A. Rodríguez-Granillo (grodriguezgranillo@gmail.com).

${ }^{2}$ Department of Pediatric Surgery, Fundación Hospitalaria, Private Children's Hospital, Buenos Aires, Argentina.

Supplemental Data

Available online at www.ajronline.org.

AJR2017; 208:1-8

0361-803X/17/2084-1

(c) American Roentgen Ray Society

OBJECTIVE. We sought to explore whether patients with pectus excavatum have exaggerated interventricular dependence and to evaluate the impact of the malformation severity (assessed on CT) on both anatomic and functional cardiac parameters (assessed on cardiac MRI).

SUBJECTS AND METHODS. The current study involved consecutive patients with a diagnosis of pectus excavatum who were referred to undergo cardiac MRI and chest CT to establish surgical candidacy or to define treatment strategies.

RESULTS. Sixty-two patients with pectus excavatum underwent cardiac MRI and chest CT. Fifty $(81 \%)$ patients were male, and the median age was 17.5 years (range, 14.0-23.0 years). Forty-seven (76\%) patients had evidence of right ventricular compression. The left ventricle showed a significantly decreased end-diastolic volume (inspiration vs expiration: $70.4 \pm 11.6$ vs $76.1 \pm 13.7 \mathrm{~mL} / \mathrm{m}^{2}$, respectively; $\left.p=0.01\right)$ and a significantly higher eccentricity index $(1.52 \pm 0.2$ vs $1.20 \pm 0.1, p<0.0001)$ during inspiration than during expiration. The median respiratory-related septal excursion was $8.1 \%$ (interquartile range, $5.1-11.7 \%$ ). Patients with pericardial effusion showed a significantly higher pectus excavatum severity index than patients without pericardial effusion $(6.3 \pm 3.4$ vs $4.4 \pm 1.3$, respectively; $p=0.003)$. Patients with a relative septal excursion equal to or larger than $11.8 \%$ showed a significantly higher pectus excavatum severity index than patients with a relative septal excursion of less than $11.8 \%(6.3 \pm 2.6$ vs $4.7 \pm 2.4$, respectively; $p=0.05)$.

CONCLUSION. In this study, patients with pectus excavatum showed significant alterations of cardiac morphology and function that were related to the deformation severity and that manifest as an exaggerated interventricular dependence.

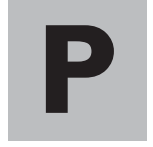
ectus excavatum is a relatively common congenital malformation of the anterior chest wall that is observed more frequently in men; it is characterized by a depression of the sternum and adjacent cartilages. Patients with pectus excavatum have a lower quality of life because of cosmetic issues that can lead to significant psychosocial impact, particularly among children and young adults, and can have impaired pulmonary functional test results [1-3]. Moreover, pectus excavatum has recently been related to decreased exercise performance and decreased cardiac function; this impairment is at least partly attributed to the compression of the right-sided chambers between the sternum and the spine $[4,5]$.

Cardiac MRI is currently the reference standard for anatomic and functional cardiac assessments, particularly for the assessment of the right ventricle (RV) [6]. Furthermore, unlike echocardiography, cardiac MRI can be used to assess surrounding structures such as the chest wall. Pathologic (incremented) interventricular dependence and the resulting ventricular septal shift to the left are typically associated with clinical conditions that interfere with pericardium distensibility, such as constrictive pericarditis. Cardiac MRI can be used to assess ventricular septal shape and motion and the extent of the excursion using real-time operator-guided breathing cine sequences [7].

Pectus excavatum is associated to some extent with external pericardial constraint. A recent echocardiographic study has shown a significant decrease in right chamber compression after surgical repair [8]. However, to our knowledge, cardiac MRI data regarding respiratory cycle changes in cardiac anatomy and function and the relationship of cardiac MRI data with thoracic indexes are lacking. Accordingly, we sought to explore whether patients with pectus excavatum have exag- 


\section{Deviggiano et al.}

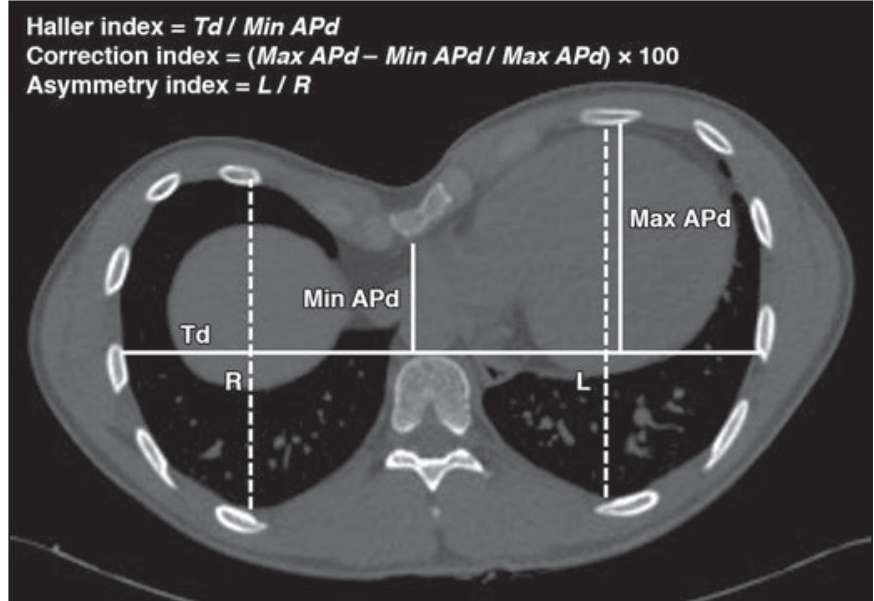

Fig. 1-CT scan of 16-year-old boy shows measurements needed to calculate thoracic indexes. $T d$ refers to transverse diameter; Min APd, minimum anteroposterior diameter; Max $A P d$, maximum anteroposterior diameter; $L$, left; and $R$, right. gerated interventricular dependence and to evaluate the impact of the malformation severity (assessed using CT) on both anatomic and functional cardiac parameters (assessed using cardiac MRI).

\section{Subjects and Methods}

This observational investigator-driven study involved consecutive patients with a diagnosis of pectus excavatum who were referred to undergo cardiac MRI and chest CT to establish surgical candidacy or to define treatment strategies. Inclusion criteria were age of more than 8 years old and the presence of Haller index of $\geq 3$ on cross-sectional imaging using CT. Patients with previous surgical correction of pectus excavatum were excluded.

All procedures performed were in accordance with the ethical standards of the institutional research committee and with the 1964 Helsinki Declaration and its later amendments. Informed consent was obtained from all participants included in the study.

\section{Chest CT Acquisition}

CT scans were obtained using a 256-MDCT scanner (ICT, Philips Healthcare). Acquisition parame-

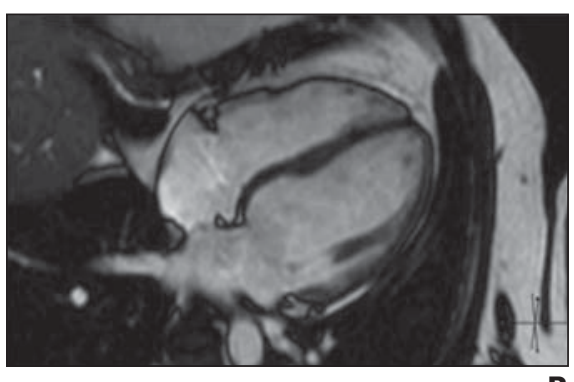

B

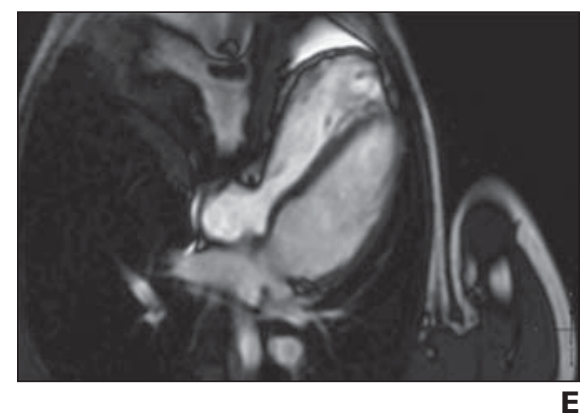

ters were the following: tube voltage, $80 \mathrm{kVp}$; tube current, $100 \mathrm{~mA}$; pitch, 0.699; collimation, $128 \times$ $0.625 \mathrm{~mm}$; and rotation time, 0.75 second. Automatic tube current dose modulation (D-DOM, Philips Medical Systems) was applied during acquisitions. Images were reconstructed at 1.0-mm-thick sections with a soft-tissue algorithm. Patients were scanned craniocaudally during a full-expiration breath-hold. IV contrast material was not administered.

\section{Cardiac MRI Acquisition}

All MRI examinations were performed on the same system (Achieva 1.5 T, Philips Healthcare) using a 5-element cardiac phased-array coil for signal reception and using a vector ECG for cardiac synchronization. Cine cardiac MRI (balanced steadystate free-precession [SSFP]) sequences were acquired at end-expiration using long-axis slices (horizontal, vertical, and left ventricle [LV] outflow tract) and 8-10 contiguous short-axis slices from the level of the mitral valve annulus through the LV apex; commercially available SSFP pulse sequences were performed, and the technical parameters were as follows: TR/TE, 3.5/1.8; flip angle, $60^{\circ}$; section thickness, $8 \mathrm{~mm}$; matrix, $144 \times 157$; FOV, $320 \mathrm{~mm}$; voxel size, $2.2 \times 2.0 \mathrm{~mm}$; and number of phases, 30. In a subset of 34 patients, the short-axis stack (8-10 slices) cine cardiac MRI examination was repeated at end-inspiration to assess volumetric variations related to the respiratory cycle.
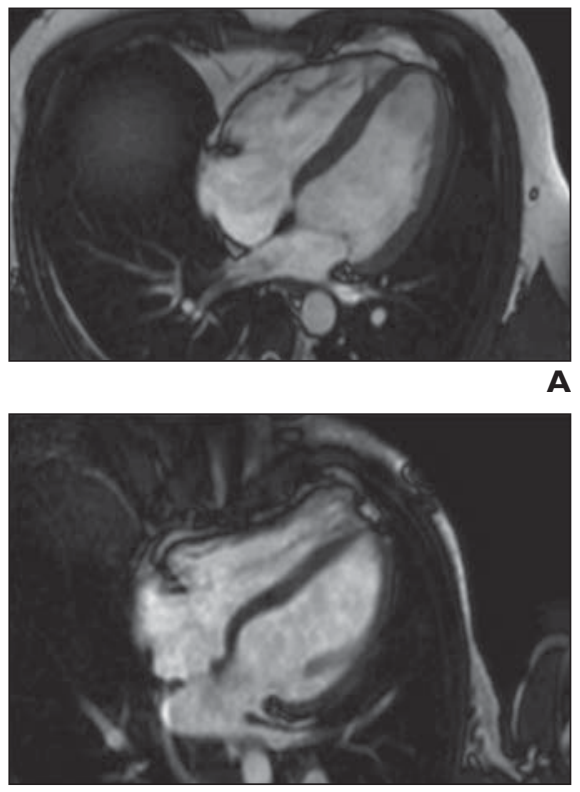

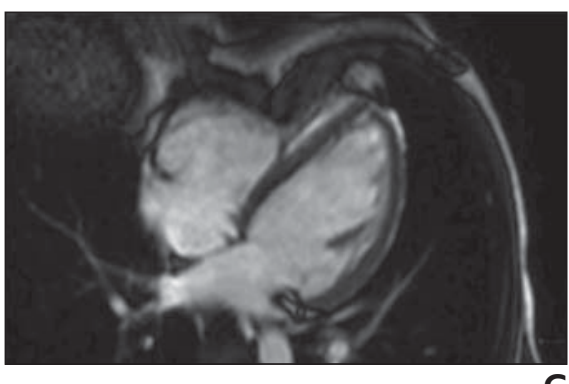

C

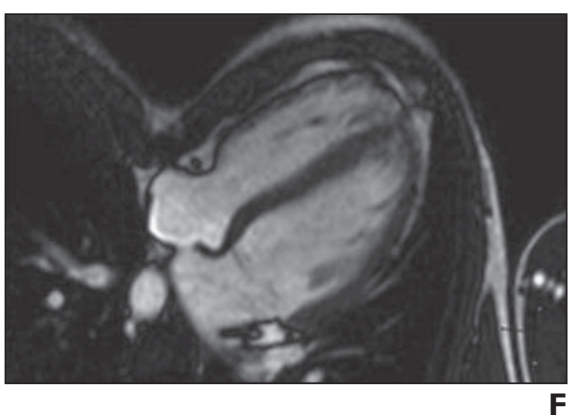

Fig. 2-Classification of right-heart compression.

A and B, Class I: no right-heart compression. MR image shows normal heart position (class la) in healthy 35 -year-old man (A), and MR image shows left-heart shift (class Ib) in 27-year-old man (B).

C and D, Class II: right ventricle (RV) compression. MR image shows mid RV compression (class Ila) in 13-year-old boy (C), and MR image shows mid and basal RV compression (class Ilb) in 13-year-old boy (D).

E, Class III: right atrioventricular groove compression. MR image shows right atrioventricular groove compression in 22-year-old woman.

F, Class IV: RV compression and atrioventricular groove compression. MR image shows RV compression and atrioventricular groove compression in 25-year-old man. 


\section{Interventricular Dependence in Pectus Excavatum}

TABLE I: Ventricular Dimensions and Atrioventricular Groove and Atrial Dimensions

\begin{tabular}{l|c}
\hline \multicolumn{1}{c|}{ Measurement } & Value \\
\hline Left ventricle & $47.5 \pm 4.1$ \\
End-diastolic diameter $(\mathrm{mm})$ & $33.1 \pm 3.3$ \\
End-systolic diameter $(\mathrm{mm})$ & $54.9 \pm 3.7$ \\
Ejection fraction $(\%)$ & $76.6 \pm 12.3$ \\
End-diastolic volume $\left(\mathrm{mL} / \mathrm{m}^{2}\right)$ & $35.0 \pm 6.2$ \\
End-systolic volume $\left(\mathrm{mL} / \mathrm{m}^{2}\right)$ & \\
Right ventricle & $50.9 \pm 6.5$ \\
Long-axis diameter $\left(\mathrm{mm} / \mathrm{m}^{2}\right)$ & $22.9 \pm 9.1$ \\
Short-axis diameter $\left(\mathrm{mm} / \mathrm{m}^{2}\right)$ & $51.1 \pm 7.0$ \\
Ejection fraction $(\%)$ & $69.4 \pm 15.0$ \\
End-diastolic volume $\left(\mathrm{mL} / \mathrm{m}^{2}\right)$ & $34.7 \pm 9.4$ \\
End-systolic volume $\left(\mathrm{mL} / \mathrm{m}^{2}\right)$ & \\
Atrioventricular groove and atrial & \\
dimensions & $15.6 \pm 3.3$ \\
Left atrium (cm $)$ & $16.5 \pm 3.8$ \\
Right atrium (cm $\left.{ }^{2}\right)$ & $28.6 \pm 4.7$ \\
Tricuspid annulus (mm) & $33.3 \pm 4.1$ \\
Mitral annulus (mm) & $0.8 \pm 0.2$ \\
Tricuspid-mitral annulus & \\
\hline
\end{tabular}

Note—Data are reported as means \pm SD.

Real-time (i.e., free-breathing, nontriggered) assessment of ventricular coupling was assessed using balanced SSFP sequences in combination with parallel imaging sequences at a basal half short ventricular axis (reconstructed pixel size, $1.15 \times 1.15 \mathrm{~mm}$ ) and a scanning time of approximately 36 seconds covering at least three complete respiratory cycles. During image acquisition, patients were instructed to breathe deeply in and out.

\section{Chest CT Analysis}

$\mathrm{CT}$ analyses were performed by an experienced radiologist blinded to the clinical data and cardiac MRI results. Two chest wall diameters were measured: a minimum anteroposterior diameter and a maximum transverse diameter. We measured both diameters at the point of maximal sternal depression. The Haller index (HI) was calculated using those variables from dividing the transverse diameter (the largest horizontal distance of the inside of the ribcage) by the anteroposterior diameter (the shorter distance between the vertebrae and the sternum) [9]. For the correction index, which corresponds to the percentage of chest depth to be corrected by bar placement, we drew a horizontal line across the anterior spine and measured two distances: the minimum distance between the posterior sternum and the anterior spine and the maximum distance between the line placed on the anterior spine and the inner margin of the most anterior portion of the chest. The difference between these two lines is the amount of defect the patient has in the chest [10] (Fig. 1).

Three cardiac diameters were also measured at the deepest point of cardiac depression: two anteroposterior (minimum and maximum) and transverse diameters as previously reported [11, 12]. The cardiac asymmetry index was calculated as reported by Kim et al. [12].

Morphologic and functional cardiac MRI variables were compared between groups according to patient stratification in HI tertiles and, as a secondary analysis, in correction index tertiles.

\section{Cardiac MRI Analysis}

All cardiac MRI studies were analyzed offline using a dedicated workstation (ViewForum, Philips Healthcare) by consensus of two similarly experienced observers ( $>6$ years of experience with cardiac MRI) who were blinded to clinical history and CT data. The presence and pattern of rightheart compression were determined on the horizontal long plane (Fig. 2).

LV end-diastolic volume (EDV) and end-systolic volume (ESV) were calculated as standardized by the Society for Cardiovascular Magnetic Resonance [13]. Basal image position was defined as the basal-most image encompassing at least $75 \%$ of the circumference of the myocardium.

For assessment of ventricular coupling, the short-axis real-time cine MR images were visually analyzed for abnormal motion of the ventricular septum throughout the respiratory cycle during early ventricular filling, as previously reported (Video S1, which can be viewed in the $A J R$ electronic supplement to this article, available at www.ajronline.org). The respiratory-related septal excursion was quantified by measuring the distance between the RV free wall and the septum as well as the biventricular diameter in a single plane (Figs. 3 and 4). During inspiration and expiration, these measurements were performed using the heartbeat that showed the largest septal displacement to the left and to the right, respectively. As reported by Francone et al. [7], normalized septal excursion (percentage septal shift) was calculated by dividing the RV wall-to-septum distance by the biventricular diameter at inspiration and expiration, respectively. We further evaluated the number of patients with a respiratory-related septal excursion equal to or larger than $11.8 \%$, the previously defined cutoff value using the mean normal value plus 2 SDs [7]. Furthermore, the LV eccentricity index (a surrogate of the extent of RV overload and septal bowing) at both expiration and inspiration was calculated at end-diastole as the superior-inferior LV diameter divided by the anterior-posterior diameter; a value of greater than 1.0 was suggestive of RV overload [14, 15] (Fig. 3).

\section{Statistical Analysis}

Discrete variables are presented as counts and percentages. Continuous variables are presented as means \pm SD and as medians (interquartile range), as indicated. Comparisons among groups were performed, as indicated, using paired samples, independent-samples $t$ test, and one-way ANOVA (with the Bonferroni test for posthoc multiple comparisons) for continuous variables with normal distribution or nonparametric tests (Wilcoxon signed rank test) for continuous variables without normal distribution. Spearman correlation coefficients were used to explore nonparametric correlations. All statistical analyses were performed using statistics software (SPSS, version 22, IBM). A two-sided $p<$ 0.05 indicated statistical significance.

\section{Results}

Sixty-two patients with pectus excavatum underwent cardiac MRI and chest CT. Fifty $(81 \%)$ patients were male; the median age was 17.5 years (range, 14.0-23.0 years). At clinical presentation, 23 (42\%) patients were asymptomatic, 16 (29\%) had atypical chest pain, and 16 (29\%) had exertional dyspnea. Clinical data were unavailable for seven patients. The mean heart rate was $72.3 \pm 12.1$ beats/min. The approximate effective radiation dose was $1.1 \mathrm{mSv}$ (dose-length product, $80 \mathrm{mGy} \times \mathrm{cm}$ ).

\section{Cardiac MRI}

All patients completed the cardiac MRI procedure, and periods of apnea were well tolerated. Mild pericardial effusion was observed in $21(34 \%)$ patients, and minimal (laminar) pleural effusion was identified in five $(8 \%)$ patients.

With regard to the type of RV compression (Fig. 2), 15 (24\%) had no RV compression (2/15 with left-side cardiac shift), whereas 47 patients had evidence of RV compression (mid RV, 22 [36\%]; mid and basal RV, 6 [10\%]; atrioventricular groove, 1 [2\%]; and atrioventricular groove and RV, 18 [29\%]).

The mean LV and RV dimensions are listed in Table 1. The mean tricuspid annulus width was $28.6 \pm 4.7 \mathrm{~mm}$, and the mean mitral annulus width was $33.3 \pm 4.1 \mathrm{~mm}$ (mean tricuspid-mitral annulus width, $0.8 \pm 0.2$ ). Among patients who underwent expiratory and inspiratory short-axis stack cine cardiac MRI $(n=34)$, a significant decrease in left 


\section{Deviggiano et al.}

ventricular dimensions was observed during the inspiratory phase (Table 2).

Real-time cine cardiac MRI of sufficient quality was performed in 55 patients. In this subset of patients, the RV short axis was significantly higher during inspiration than expiration $(26.6 \pm 8.7$ vs $24.9 \pm 6.3 \mathrm{~mm}$, respectively; $p=0.03)$. Likewise, the LV eccentricity index was significantly higher during inspiration than expiration $(1.52 \pm 0.2$ vs $1.20 \pm 0.1, p<0.0001)$. This difference was associated with a significantly higher superior-inferior LV diameter during inspiration $(61.3 \pm 5.8$ vs $56.4 \pm 5.8 \mathrm{~mm}, p<0.0001)$. Furthermore, the median respiratory-related septal excursion was $8.1 \%$ (interquartile range, 5.1-11.7\%), and 13 of 55 (24\%) patients had a respiratory-related septal excursion equal to or larger than $11.8 \%$.

\section{CT Thoracic Indexes}

The mean thoracic indexes of the study population were as follows: $\mathrm{HI}, 5.1 \pm 2.4$; correction index, $34.2 \% \pm 17.6 \%$; and cardiac asymmetry index, $1.5 \pm 0.5$. Tables 3 and 4 show cardiac morphologic and functional findings according to the HI and correction index tertiles. Patients with pericardial effusion, although mild, showed significantly higher HI values $(6.3 \pm 3.4$ vs $4.4 \pm 1.3$, respectively; $p=0.003$ ) and trends toward higher correction index values $(40.2 \% \pm$ $22.5 \%$ vs $31.8 \% \pm 12.7 \%, p=0.07)$ and higher cardiac asymmetry index values $(1.70 \pm$ 0.8 vs $1.45 \pm 0.3, p=0.07$ ) than patients without pericardial effusion. Likewise, patients with a relative septal excursion equal to or larger than $11.8 \%$ showed significantly higher HI values $(6.3 \pm 2.6$ vs $4.7 \pm 2.4$, respectively; $p=0.05)$ and correction index values $(47.8 \% \pm 16.4 \%$ vs $31.7 \% \pm 15.2 \%, p=0.003)$ than patients with septal excursion smaller than $11.8 \%$.

Significant positive correlations were identified between respiratory-related septal excursion and the HI $(r=0.37, p=0.07)$, correction index ( $r=0.46, p<0.0001)$, and cardiac asymmetry index $(r=0.49, p<0.001)$. Significant correlations, albeit weaker, between the eccentricity index and the HI $(r=$ $0.27, p=0.049)$, correction index $(r=0.34$, $p=0.01)$, and cardiac asymmetry index $(r=$ $0.37, p=0.007)$ were also shown. Similarly, significant inverse relationships were observed between the tricuspid annulus width and the HI $(r=-0.34, p=0.01)$, correction index $(r=-0.28, p=0.35)$, and cardiac asymmetry index $(r=-0.36, p=0.006)$.

TABLE 2: Ventricular Dimensions Among Patients Who Underwent Expiratory and Inspiratory Short-Axis Stack Cine Cardiac MRI

\begin{tabular}{l|c|c|c}
\hline \multicolumn{1}{c|}{ Measurement } & Expiration & Inspiration & $p$ \\
\hline Left ventricle & & & \\
End-diastolic diameter (mm) & $47.8 \pm 4.0$ & $44.9 \pm 0.7$ & $<0.0001$ \\
Ejection fraction (\%) & $54.5 \pm 4.3$ & $55.9 \pm 4.5$ & 0.02 \\
End-diastolic volume (mL/m²) & $76.1 \pm 13.7$ & $70.4 \pm 11.6$ & 0.01 \\
End-systolic volume $\left(\mathrm{mL} / \mathrm{m}^{2}\right)$ & $35.4 \pm 6.9$ & $31.1 \pm 6.5$ & $<0.0001$ \\
Right ventricle & & & \\
Long-axis diameter $(\mathrm{mm})$ & $51.9 \pm 5.8$ & $52.3 \pm 6.1$ & 0.39 \\
Short-axis diameter $(\mathrm{mm})$ & $23.0 \pm 11.0$ & $19.3 \pm 5.4$ & 0.04 \\
Ejection fraction $(\%)$ & $53.6 \pm 7.2$ & $54.0 \pm 15.6$ & 0.88 \\
End-diastolic volume $\left(\mathrm{mL} / \mathrm{m}^{2}\right)$ & $73.3 \pm 12.5$ & $68.5 \pm 13.8$ & 0.003 \\
End-systolic volume $\left(\mathrm{mL} / \mathrm{m}^{2}\right)$ & $34.1 \pm 8.5$ & $33.2 \pm 9.0$ & 0.32 \\
\hline
\end{tabular}

Note-Data are reported as means \pm SD.

TABLE 3: Morphologic and Functional Cardiac MRI Data After Stratification in Haller Index (HI) Tertiles

\begin{tabular}{|c|c|c|c|c|}
\hline \multirow[b]{2}{*}{ Measurement } & \multicolumn{3}{|c|}{$\mathrm{HI}$} & \multirow[b]{2}{*}{ ANOVA } \\
\hline & First Tertile & Second Tertile & Third Tertile & \\
\hline Tricuspid annulus width (mm) & $30.8 \pm 3.9$ & $28.2 \pm 4.5$ & $26.6 \pm 5.2^{\mathrm{a}}$ & 0.02 \\
\hline Mitral annulus width (mm) & $35.1 \pm 3.3$ & $32.3 \pm 4.2$ & $32.9 \pm 4.6$ & 0.10 \\
\hline Tricuspid-annulus ratio & $0.88 \pm 0.1$ & $0.88 \pm 0.1$ & $0.81 \pm 0.2$ & 0.22 \\
\hline Left atrium area $\left(\mathrm{cm}^{2}\right)$ & $17.0 \pm 2.9$ & $15.7 \pm 3.1$ & $14.2 \pm 3.5^{\mathrm{a}}$ & 0.04 \\
\hline Right atrium area $\left(\mathrm{cm}^{2}\right)$ & $17.3 \pm 3.5$ & $16.8 \pm 3.8$ & $15.5 \pm 4.2$ & 0.34 \\
\hline \multicolumn{5}{|l|}{ Left ventricle } \\
\hline Septal excursion (\%), median (IQR) & $6.2(3.3-9.0)$ & $7.5(5.6-10.7)$ & $10.9(7.8-14.1)$ & $<0.0001^{b}$ \\
\hline Eccentricity index (expiration) & $1.16 \pm 0.1$ & $1.20 \pm 0.1$ & $1.22 \pm 0.1$ & 0.08 \\
\hline Eccentricity index (inspiration) & $1.46 \pm 0.2$ & $1.52 \pm 0.2$ & $1.58 \pm 0.2$ & 0.20 \\
\hline End-diastolic diameter (mm) & $49.8 \pm 4.2$ & $45.9 \pm 3.9$ & $46.9 \pm 3.3$ & 0.007 \\
\hline End-systolic diameter (mm) & $34.6 \pm 3.7$ & $32.2 \pm 3.1$ & $32.7 \pm 2.8$ & 0.07 \\
\hline End-diastolic volume $\left(\mathrm{mL} / \mathrm{m}^{2}\right)$ & $78.6 \pm 10.6$ & $74.6 \pm 10.7$ & $75.9 \pm 15.4$ & 0.57 \\
\hline End-systolic volume $\left(\mathrm{mL} / \mathrm{m}^{2}\right)$ & $36.5 \pm 5.9$ & $32.5 \pm 5.1$ & $36.6 \pm 7.1$ & 0.047 \\
\hline Stroke volume $\left(\mathrm{mL} / \mathrm{m}^{2}\right)$ & $42.0 \pm 7.0$ & $42.0 \pm 6.8$ & $42.2 \pm 4.3$ & 0.99 \\
\hline Ejection fraction (\%) & $53.9 \pm 4.2$ & $56.4 \pm 3.2$ & $53.8 \pm 3.3$ & 0.03 \\
\hline \multicolumn{5}{|l|}{ Right ventricle } \\
\hline Short-axis diameter $\left(\mathrm{mm} / \mathrm{m}^{2}\right)$ & $27.4 \pm 14.6$ & $22.4 \pm 4.7$ & $19.6 \pm 4.5^{\mathrm{a}}$ & 0.03 \\
\hline Long-axis diameter $\left(\mathrm{mm} / \mathrm{m}^{2}\right)$ & $49.2 \pm 8.9$ & $51.7 \pm 4.8$ & $51.4 \pm 6.0$ & 0.47 \\
\hline End-diastolic volume $\left(\mathrm{mL} / \mathrm{m}^{2}\right)$ & $68.4 \pm 16.4$ & $68.1 \pm 10.1$ & $71.1 \pm 18.7$ & 0.80 \\
\hline End-systolic volume $\left(\mathrm{mL} / \mathrm{m}^{2}\right)$ & $36.2 \pm 9.6$ & $33.1 \pm 5.6$ & $34.7 \pm 12.8$ & 0.60 \\
\hline Ejection fraction (\%) & $51.1 \pm 6.6$ & $50.8 \pm 7.1$ & $51.7 \pm 7.7$ & 0.93 \\
\hline
\end{tabular}

Note-Unless indicated otherwise, data are reported as means $\pm S D$. IQR = interquartile range.

${ }^{a}$ For first tertile versus third tertile (Bonferroni), $p<0.05$.

bWilcoxon signed rank test.

Left atrium area (vs HI: $r=-0.34, p=0.009$; vs correction index: $r=-0.50, p<0.0001$; vs cardiac asymmetry index: $r=-0.32, p=$ $0.01)$ and right atrium area (vs HI: $r=-0.20$, $p=0.12$; vs correction index: $r=-0.32, p=$ 0.01 ; vs cardiac asymmetry index: $r=-0.11$, $p=0.44$ ) were inversely correlated to thoracic indexes. 


\section{Interventricular Dependence in Pectus Excavatum}

TABLE 4: Morphologic and Functional Cardiac MRI Data After Stratification in Correction Index Tertiles

\begin{tabular}{|c|c|c|c|c|}
\hline \multirow[b]{2}{*}{ Data } & \multicolumn{3}{|c|}{ Correction Index (\%) } & \multirow[b]{2}{*}{ ANOVA } \\
\hline & First Tertile & Second Tertile & Third Tertile & \\
\hline Tricuspid annulus width (mm) & $29.5 \pm 4.8$ & $29.3 \pm 4.8$ & $26.6 \pm 4.5$ & 0.12 \\
\hline Mitral annulus width (mm) & $34.6 \pm 4.0$ & $32.9 \pm 4.4$ & $32.6 \pm 4.0$ & 0.28 \\
\hline Tricuspid-annulus ratio & $0.86 \pm 0.1$ & $0.89 \pm 0.1$ & $0.83 \pm 0.2$ & 0.44 \\
\hline Left atrium area $\left(\mathrm{cm}^{2}\right)$ & $17.2 \pm 2.7$ & $15.7 \pm 3.1$ & $13.9 \pm 3.5^{\mathrm{a}}$ & 0.007 \\
\hline Right atrium area $\left(\mathrm{cm}^{2}\right)$ & $17.5 \pm 3.5$ & $16.9 \pm 3.6$ & $15.1 \pm 4.3$ & 0.13 \\
\hline \multicolumn{5}{|l|}{ Left ventricle } \\
\hline Septal excursion (\%), median (IQR) & $5.1(3.0-8.1)$ & $7.6(5.1-10.2)$ & $11.6(7.9-14.4)$ & $<0.0001^{b}$ \\
\hline Eccentricity index (expiration) & $1.16 \pm 0.1$ & $1.20 \pm 0.1$ & $1.22 \pm 0.1$ & 0.07 \\
\hline Eccentricity index (inspiration) & $1.43 \pm 0.2$ & $1.54 \pm 0.2$ & $1.58 \pm 0.2$ & 0.09 \\
\hline End-diastolic diameter (mm) & $48.5 \pm 4.6$ & $46.7 \pm 3.9$ & $46.9 \pm 3.5$ & 0.30 \\
\hline End-systolic diameter (mm) & $34.4 \pm 3.8$ & $32.3 \pm 3.0$ & $32.8 \pm 2.8$ & 0.10 \\
\hline End-diastolic volume $\left(\mathrm{mL} / \mathrm{m}^{2}\right)$ & $76.3 \pm 10.9$ & $75.8 \pm 10.0$ & $76.6 \pm 16.0$ & 0.98 \\
\hline End-systolic volume $\left(\mathrm{mL} / \mathrm{m}^{2}\right)$ & $35.2 \pm 6.4$ & $33.2 \pm 4.9$ & $36.7 \pm 7.2$ & 0.21 \\
\hline Stroke volume $\left(\mathrm{mL} / \mathrm{m}^{2}\right)$ & $41.0 \pm 6.5$ & $42.5 \pm 6.5$ & $42.8 \pm 5.1$ & 0.64 \\
\hline Ejection fraction (\%) & $54.2 \pm 4.0$ & $56.1 \pm 3.4$ & $54.0 \pm 3.4$ & 0.13 \\
\hline \multicolumn{5}{|l|}{ Right ventricle } \\
\hline Short-axis diameter $\left(\mathrm{mm} / \mathrm{m}^{2}\right)$ & $25.8 \pm 14.1$ & $23.6 \pm 4.5$ & $19.4 \pm 5.2$ & 0.09 \\
\hline Long-axis diameter $\left(\mathrm{mm} / \mathrm{m}^{2}\right)$ & $49.4 \pm 8.8$ & $51.3 \pm 4.1$ & $51.8 \pm 6.3$ & 0.49 \\
\hline End-diastolic volume $\left(\mathrm{mL} / \mathrm{m}^{2}\right)$ & $65.1 \pm 15.4$ & $71.0 \pm 11.0$ & $71.3 \pm 17.8$ & 0.34 \\
\hline End-systolic volume $\left(\mathrm{mL} / \mathrm{m}^{2}\right)$ & $34.3 \pm 8.4$ & $34.7 \pm 7.5$ & $34.6 \pm 12.4$ & 0.99 \\
\hline Ejection fraction (\%) & $50.8 \pm 6.1$ & $50.9 \pm 7.5$ & $51.9 \pm 7.0$ & 0.89 \\
\hline
\end{tabular}

Note-Unless indicated otherwise, data are reported as means \pm SD. IQR $=$ interquartile range.

aFor first tertile versus third tertile (Bonferroni), $p<0.05$.

bWilcoxon signed rank test.

\section{Discussion}

The main finding of our study was that alterations of cardiac morphology and function in patients with pectus excavatum are relat- ed to the severity of the deformation and that these alterations manifest during inspiration.

Only 2 decades ago, pectus excavatum was deemed a cosmetic condition, and the clinical symptoms of patients with this condition were underrecognized or were considered to not be related to pectus excavatum [16]. Indeed, although pectus excavatum has been associated to abnormal cardiopulmonary function test results, there is lack of consensus regarding whether chest deformity can cause symptoms and abnormal functional test results of sufficient severity to justify surgery $[17,18]$. Nevertheless, surgical correction has rapidly gained acceptance during the past decade, and minimally invasive repair has become a safe standard for the treatment of patients with pectus excavatum; excellent results have been reported in most patients, and positive impacts on quality of life and cardiopulmonary function have been observed $[8,18,19]$.

Accordingly, it is essential to establish the impact of the severity of the deformation on cardiac anatomy and function, particularly among children and young adults because the surgical decision is challenging in these patients given the intraoperative risk and the relatively variable rates of infection, bar migration, and reoperation [20-22].

Previous studies using cardiac MRI for the assessment of patients with pectus excavatum have reported distorted RV geometry and reduced RV ejection fraction [23, 24]. To our knowledge, our study is the first to explore respiratory cycle changes in cardiac anatomy and function values and the relationship of those values with pectus excavatum indexes. In this study, significant changes in both cardiac chamber dimensions and function were observed in relation to the respiratory cycle. In particular, an exaggerated interventricular dependence was identified

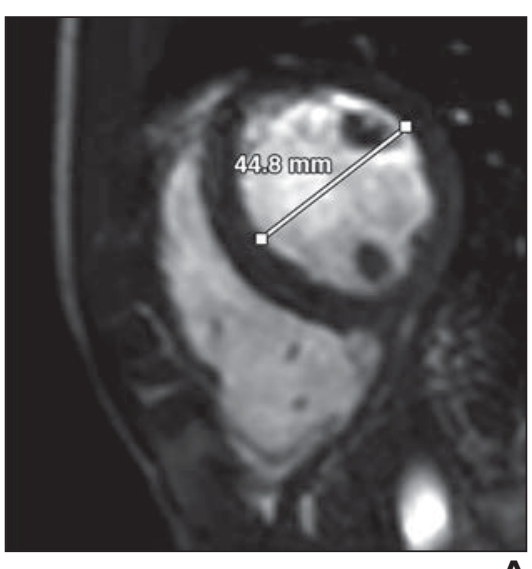

A
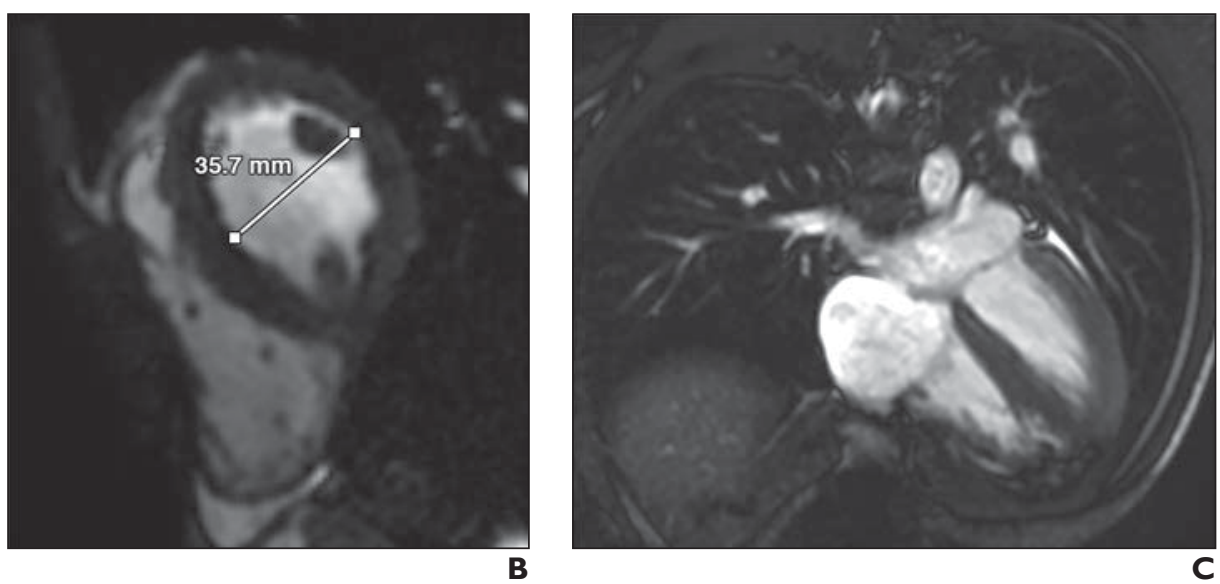

Fig. 3-15-year-old boy with Haller index of 7.2, correction index of 54\%, cardiac asymmetry index of 1.6, and class IV right-heart compression.

$\mathbf{A}$ and B, Balanced steady-state free-precession images obtained at mid short axis during expiration (A) and inspiration (B) show dimensions of left ventricle (LV) are smaller during inspiration (line, B: $35.7 \mathrm{~mm}$ ) than expiration (line, A: $44.8 \mathrm{~mm}$ ). LV end-diastolic volume is $80 \mathrm{~mL} / \mathrm{m}^{2}$ during expiration and $62 \mathrm{~mL} / \mathrm{m}^{2}$ during inspiration. C, End-systole cine cardiac MR image obtained at horizontal long axis.

(Fig. 3 continues on next page) 


\section{Deviggiano et al.}
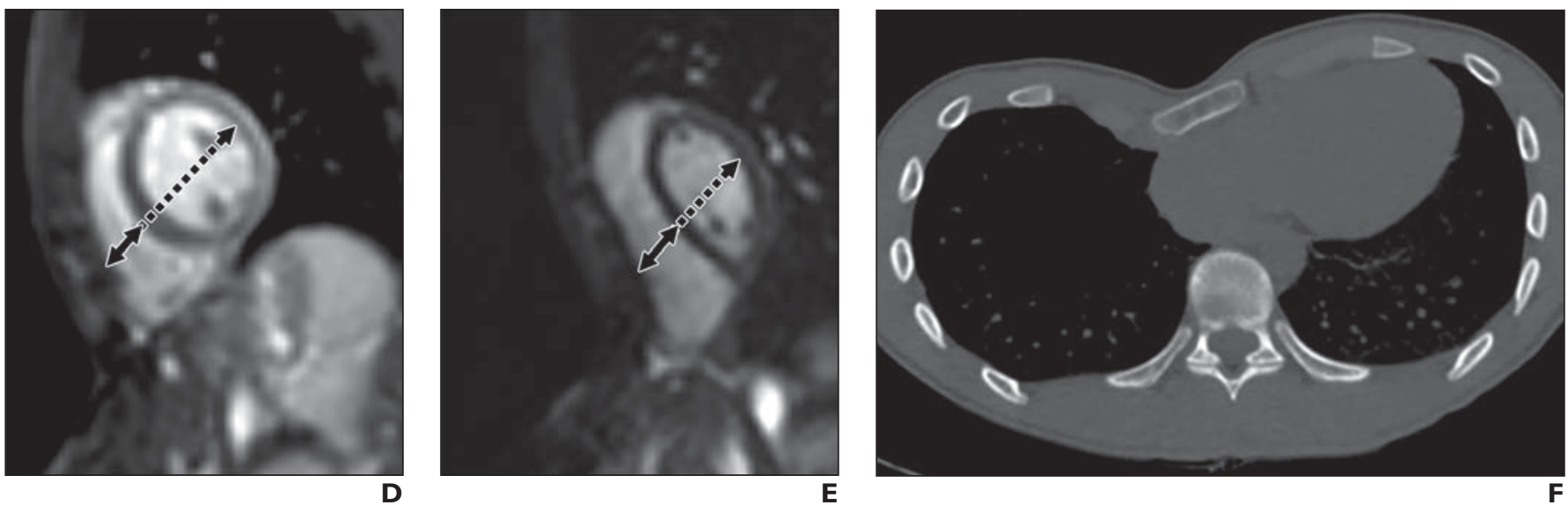

Fig. 3 (continued) - 15-year-old boy with Haller index of 7.2, correction index of $54 \%$, cardiac asymmetry index of 1.6, and class IV right-heart compression. $\mathbf{D}$ and $\mathbf{E}$, Real-time cine cardiac MR images obtained during expiration (D) and inspiration (E) show relative septal excursion of $15 \%$ and eccentricity index during inspiration of 1.9. Relative septal excursion is calculated by dividing distance between free wall of right ventricle and septum (solid arrows) by biventricular distance (dotted arrows) at early ventricular filling during inspiration and expiration. Relative septal position is $47.3 \%$ at expiration and $22.4 \%$ at inspiration, resulting in relative septal excursion of $24.9 \%$.

F, Axial chest CT image obtained at maximum compression site.

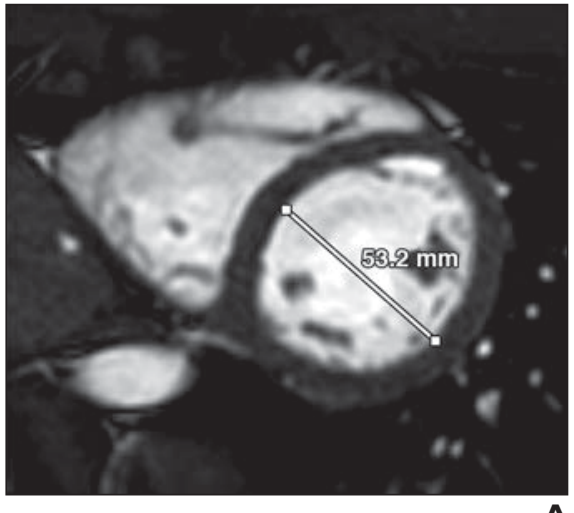

A

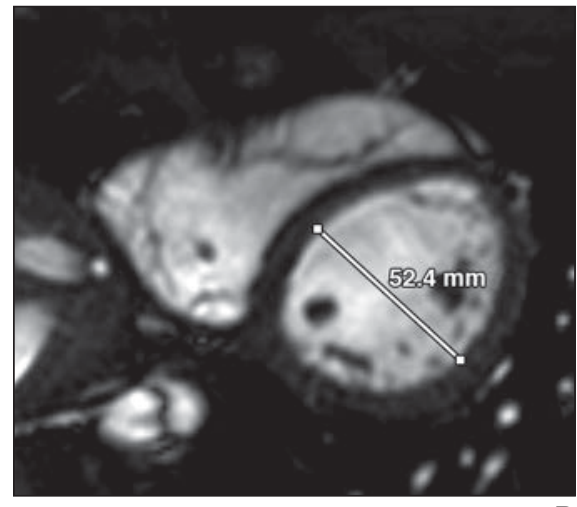

B
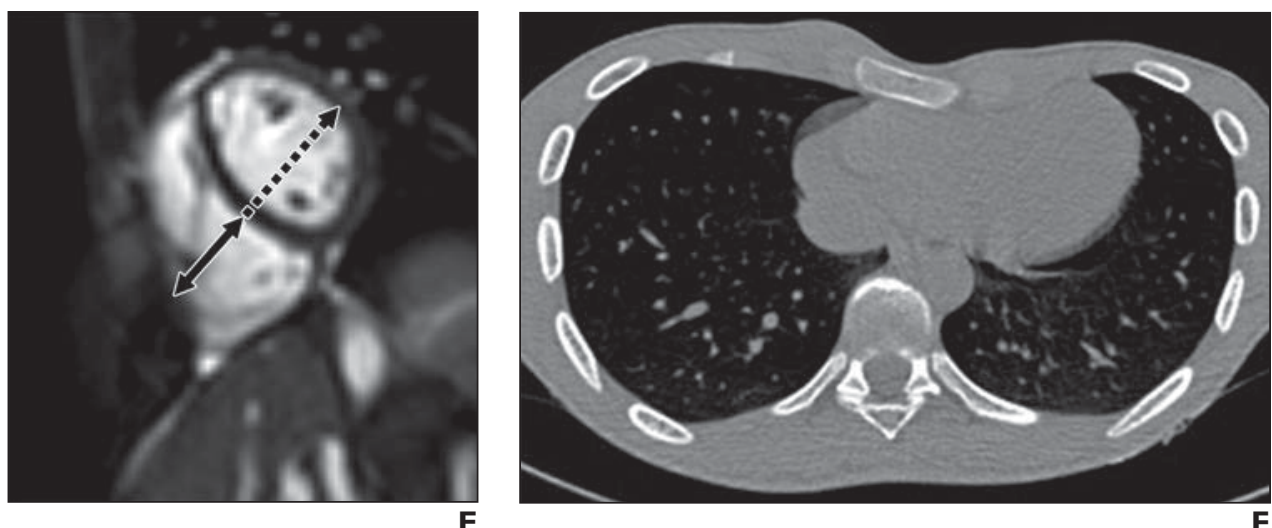

Fig. 4-15-year-old boy with Haller index of 4.8, correction index of 33\%, cardiac asymmetry index of 1.3, and class Ila right-heart compression.

$\mathbf{A}$ and $\mathbf{B}$, Balanced steady-state free-precession images obtained at mid short axis during expiration (A) and inspiration (B) show dimensions of left ventricle (LV) are similar during inspiration (line, B: $52.4 \mathrm{~mm}$ ) and expiration (line, A: $53.2 \mathrm{~mm}$ ). LV end-diastolic volume is $96 \mathrm{~mL} / \mathrm{m}^{2}$ during expiration and $94 \mathrm{~mL} / \mathrm{m}^{2}$ during inspiration.

C, End-systole cine cardiac MR image obtained at horizontal long axis.

D and E, Real-time cine cardiac MR images obtained during expiration (D) and inspiration (E) show relative septal excursion of $2 \%$ and eccentricity index during inspiration of 1.4. Relative septal excursion is calculated by dividing distance between free wall of right ventricle and septum (solid arrows) by biventricular distance (dotted arrows) at early ventricular filling during inspiration and expiration.

F, Axial chest CT image obtained at maximum compression site. 


\section{Interventricular Dependence in Pectus Excavatum}

independent of the thoracic indexes explored. This interventricular dependence was shown both by a significant respiratory-related septal excursion and by a markedly increased LV eccentricity index. It is noteworthy that $24 \%$ of the patients with pectus excavatum had a respiratory-related septal excursion equal to or larger than $11.8 \%$, the previously defined cutoff value used to discriminate between healthy patients and those with constrictive pericarditis [7]. In parallel, the LV eccentricity index, a surrogate of RV overload, was abnormal even during expiration; among the patients with a more severe chest deformation, the superior-inferior diameter was, on average, more than $50 \%$ larger than the anteriorposterior diameter. Furthermore, in line with the study of Oezcan et al. [25], 34\% of patients with pectus excavatum had evidence of mild pericardial effusion, which was related to the deformation severity. Pectus excavatum severity was also inversely related to the width of the tricuspid annulus and to the area of the left atrium. Atrioventricular groove compression has been proposed as a potential mechanism associated with reduced RV cardiac output in these patients. Furthermore, the negative correlation between left atrium area and pectus excavatum severity is consistent with the findings reported in a previous study: Humphries et al. [26] found compression relief in a substantial percentage of patients after sternal eversion. However, the potential physiologic or clinical implications of these findings are poorly understood $[8,26]$.

On the contrary, in opposition to a previous study that showed a significant increase in LV ejection fraction on echocardiography after surgical correction, we did not identify a relationship between pectus excavatum severity and this variable [27].

Our study adds to the increasing evidence supporting the potential cardiac anatomic and functional implications of pectus excavatum beyond the merely cosmetic issues. We found that pectus excavatum leads to a cardiac functional impact that is related to the deformation severity and that has a pattern similar to, albeit obviously significantly milder than, constrictive pericarditis [28]. Given the compression of the heart (also commonly displaced to the left) between the posterior chest wall indentation and the spine, a constrictivelike hemodynamic pattern is likely because of a "virtually" encased pericardium. This pattern might partially explain the paradoxical respiratory-related discordance between chest deformity (attenuating during inspiration) and cardiac function impact (attenuating during expiration).

A number of limitations should be acknowledged. The relatively small sample size might lead to selection bias, although it is worth mentioning that one of the strengths of our study is that thoracic indexes were assessed using chest CT. Given the young age of the patients included, an age-matched control group was not available. Nevertheless, in an adult population, Francone et al. [7] have previously reported that the mean respiratory-related septal excursion of healthy patients was $7.0 \% \pm 2.4 \%$, similar to the one we observed among patients at the lowest HI tertile. We do not have follow-up data for these patients regarding eventual surgical correction. Finally, short-axis cine cardiac MRI in both expiration and inspiration was performed for paired respiratory-related volumetric comparisons in only approximately half of the patients.

\section{Conclusion}

In this study, patients with pectus excavatum showed significant alterations of cardiac morphology and function that were related to the severity of the deformation and that manifest as an exaggerated interventricular dependence.

\section{References}

1. Ji Y, Liu W, Chen S, et al. Assessment of psychosocial functioning and its risk factors in children with pectus excavatum. Health Qual Life Outcomes 2011; 9:28

2. Steinmann C, Krille S, Mueller A, Weber P, Reingruber B, Martin A. Pectus excavatum and pectus carinatum patients suffer from lower quality of life and impaired body image: a control group comparison of psychological characteristics prior to surgical correction. Eur J Cardiothorac Surg 2011; 40:1138-1145

3. Maagaard M, Tang M, Ringgaard S, et al. Normalized cardiopulmonary exercise function in patients with pectus excavatum three years after operation. Ann Thorac Surg 2013; 96:272-278

4. Castellani C, Windhaber J, Schober PH, Hoellwarth ME. Exercise performance testing in patients with pectus excavatum before and after Nuss procedure. Pediatr Surg Int 2010; 26:659-663

5. Sigalet DL, Montgomery M, Harder J. Cardiopulmonary effects of closed repair of pectus excavatum. J Pediatr Surg 2003; 38:380-385

6. Geva T. Is MRI the preferred method for evaluating right ventricular size and function in patients with congenital heart disease? MRI is the preferred method for evaluating right ventricular size and function in patients with congenital heart dis- ease. Circ Cardiovasc Imaging 2014; 7:190-197

7. Francone M, Dymarkowski S, Kalantzi M, Rademakers FE, Bogaert J. Assessment of ventricular coupling with real-time cine MRI and its value to differentiate constrictive pericarditis from restrictive cardiomyopathy. Eur Radiol 2006; 16:944-951

8. Chao CJ, Jaroszewski DE, Kumar PN, et al. Surgical repair of pectus excavatum relieves right heart chamber compression and improves cardiac output in adult patients: an intraoperative transesophageal echocardiographic study. Am J Surg 2015; 210:1118-1124

9. Haller JA Jr, Scherer LR, Turner CS, Colombani PM. Evolving management of pectus excavatum based on a single institutional experience of 664 patients. Ann Surg 1989; 209:578-582

10. St. Peter SD, Juang D, Garey CL, et al. A novel measure for pectus excavatum: the correction index. J Pediatr Surg 2011; 46:2270-2273

11. Kim HC, Park HJ, Ham SY, et al. Development of automatized new indices for radiological assessment of chest-wall deformity and its quantitative evaluation. Med Biol Eng Comput 2008; 46:815-823

12. Kim M, Lee KY, Park HJ, et al. Development of new cardiac deformity indexes for pectus excavatum on computed tomography: feasibility for preand post-operative evaluation. Yonsei Med J 2009; 50:385-390

13. Schulz-Menger J, Bluemke DA, Bremerich J, et al. Standardized image interpretation and post processing in cardiovascular magnetic resonance: Society for Cardiovascular Magnetic Resonance (SCMR) board of trustees task force on standardized post processing. J Cardiovasc Magn Reson 2013; 15:35

14. Abd El Rahman MY, Hui W, Dsebissowa F, et al. Quantitative analysis of paradoxical interventricular septal motion following corrective surgery of tetralogy of Fallot. Pediatr Cardiol 2005; 26:379-384

15. Méndez C, Soler R, Rodriguez E, et al. Magnetic resonance imaging of abnormal ventricular septal motion in heart diseases: a pictorial review. Insights Imaging 2011; 2:483-492

16. Crump HW. Pectus excavatum. Am Fam Physician 1992; 46:173-179

17. Malek MH, Berger DE, Marelich WD, Coburn JW, Beck TW, Housh TJ. Pulmonary function following surgical repair of pectus excavatum: a meta-analysis. Eur J Cardiothorac Surg 2006; 30:637-643

18. Hoksch B, Kocher G, Vollmar P, Praz F, Schmid RA. Nuss procedure for pectus excavatum in adults: long-term results in a prospective observational study. Eur J Cardiothorac Surg 2016; 50:934-939

19. Jaroszewski DE, Ewais MM, Chao CJ, et al. Success of minimally invasive pectus excavatum procedures (modified Nuss) in adult patients $(\geq 30$ years). Ann Thorac Surg 2016; 102:993-1003

20. Gibreel W, Zendejas B, Joyce D, Moir CR, Zar- 


\section{Deviggiano et al.}

roug AE. Minimally invasive repairs of pectus excavatum: surgical outcomes, quality of life, and predictors of reoperation. J Am Coll Surg 2016; 222:245-252

21. Obermeyer RJ, Godbout E, Goretsky MJ, et al. Risk factors and management of Nuss bar infections in 1717 patients over 25 years. J Pediatr Surg 2016; 51:154-158

22. Fibla JJ, Molins L, Moradiellos J, et al. Experience with the Nuss technique for the treatment of pectus excavatum in Spanish thoracic surgery departments. Cir Esp 2016; 94:38-43

23. Topper A, Polleichtner S, Zagrosek A, et al.
Impact of surgical correction of pectus excavatum on cardiac function: insights on the right ventricle-a cardiovascular magnetic resonance study. Interact Cardiovasc Thorac Surg 2016; 22:38-46

24. Saleh RS, Finn JP, Fenchel M, et al. Cardiovascular magnetic resonance in patients with pectus excavatum compared with normal controls. J Cardiovasc Magn Reson 2010; $12: 73$

25. Oezcan S, Attenhofer Jost CH, Pfyffer M, et al. Pectus excavatum: echocardiography and cardiac MRI reveal frequent pericardial effusion and right-sided heart anomalies. Eur Heart $J$ Cardiovasc Imaging 2012; 13:673-679
26. Humphries CM, Anderson JL, Flores JH, Doty JR. Cardiac magnetic resonance imaging for perioperative evaluation of sternal eversion for pectus excavatum. Eur J Cardiothorac Surg 2013; 43:1110-1113

27. Krueger T, Chassot PG, Christodoulou M, Cheng C, Ris HB, Magnusson L. Cardiac function assessed by transesophageal echocardiography during pectus excavatum repair. Ann Thorac Surg 2010; 89:240-243

28. Iannucci GJ, Slesnick TC, Kogon B, Samai C. Lower extremity edema in a child due to pectus excavatum. Ann Thorac Surg 2015; 99:e29-e30

\section{FOR YOUR INFORMATION}

A data supplement for this article can be viewed in the online version of the article at: www.ajronline.org. 